\title{
Design, fabrication and testing of electroadhesive interdigital electrodes
}

https://doi.org/10.1515/phys-2018-0059

Received November 5, 2017; accepted November 12, 2017

Abstract: Electrostatic adhesion force is analyzed with emphasis on design parameters of the interdigital electrodes, material properties of dielectric layers and its thickness. From these results, two fabrication processes of the electroadhesive foils are studied to reach the highest possible performance. Experimental measurements are carried out to verify the results.

Keywords: electrostatic adhesion, silicon, stamping, carbon powder, aerosol jet printing, numerical analysis

PACS: $41.20 . \mathrm{Cv}, 68.35 . \mathrm{Np}, 81.20 . \mathrm{Rg}$

\section{Motivation and introduction}

The aim of this work is to develop light weight soft-robotic gripper system with emphasis on small size and efficiency for miniature robotic applications. There is no gripper system in use for this kind of application and electrostatic adhesion seems to the be possible solution that will help to solve more complex tasks with miniature robots. A basic idea of discussed gripper utilization is illustrated in Figure 1 in the case of magnetically guided robots with permanent magnets (PMs) [1].

\subsection{Theoretical background}

The proposed system uses interdigital electrodes that are placed between two thin dielectric layers (see Figure 2,

Jan Fessl: Department of Theory of Electrical Engineering, University of West Bohemia, Czech Republic, E-mail: jan.fess195@gmail.com

*Corresponding Author: František Mach: Department of Theory of Electrical Engineering, University of West Bohemia, Czech Republic, E-mail: fmach@rice.zcu.cz

Jiřı Navrátil: Department of Technologies and Measurement, University of West Bohemia, Czech Republic, E-mail: jnavratil@rice.zcu.cz

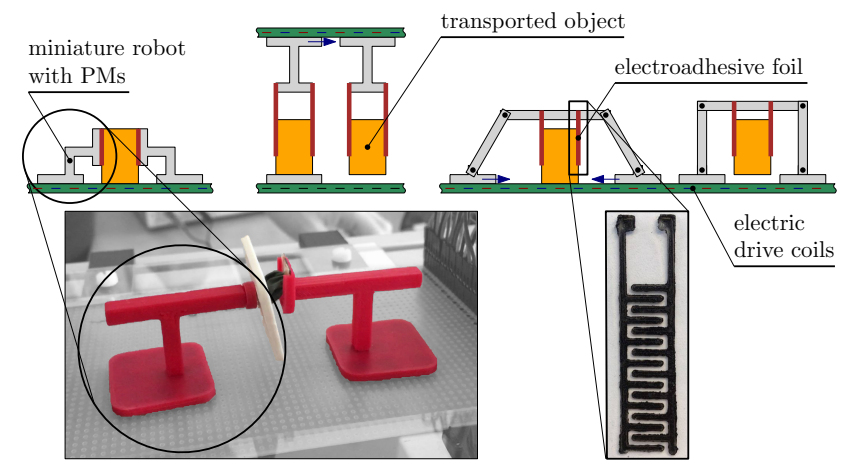

Figure 1: First ideas of grippers utilization in the case of magnetically guided actuation of miniature robots. Illustration shows three different ideas and also photos of fabricated grippers for developed system MagSnail [2]

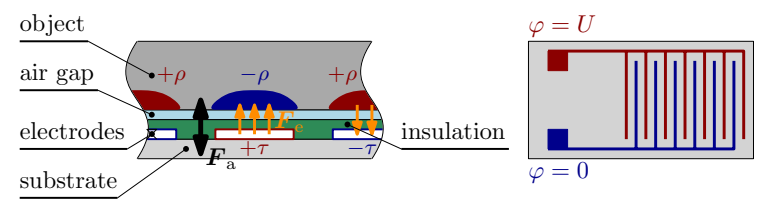

Figure 2: Basic principle of electrostatic adhesion for dielectric object. Left figure shows cross-section of electroadhesive foil with interdigital electodes and right figure top view of the foil

left). One of the electrodes is connected to the high voltage source $U$ and the other one has potential equal to zero. The electric field that is generated by electrodes can exert adhesive force to various materials. The resulting attractive force $\boldsymbol{F}_{\mathrm{a}}$ is a sum of forces $\boldsymbol{F}_{\mathrm{e}}$ that occur between the region of charges $\pm \rho$ in the manipulated materials and electrodes that have opposite charge $\pm \tau$. The gripping principle is used without any mechanical damage or chemical reaction $[3,4]$.

\subsection{Design and fabrication}

Two different fabrication approaches of electroadhesive foils were studied. The first one is based on Aerosol Jet 


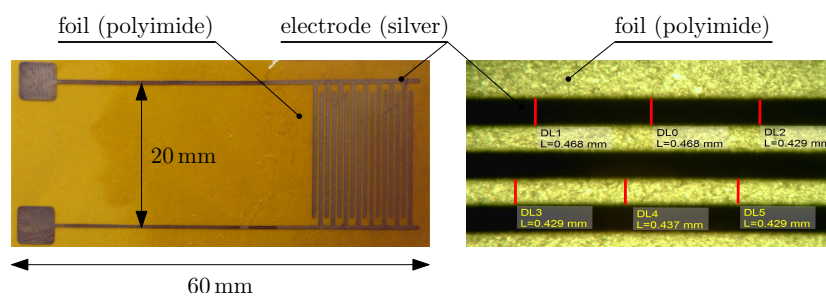

Figure 3: First prototype of flexible electroadhesive foil fabricated by AJP (left figure shows fabricated foil, right figure shows details captured by microscope)

Printing (AJP) ${ }^{1}$. AJP silver nanoparticle ink is patterned on polyimide (Kapton ${ }^{\circledR}$, DuPont). Then the nanoparticle ink was cured in the oven. A thin dielectric layer was then applied over electrodes using silicon (Ecoflex 00-30) in the final step. The most advanced prototype has excellent electrical properties (high breakdown voltage, critical electric field $E_{\mathrm{c}}=236 \mathrm{kV} \cdot \mathrm{mm}^{-1}$ ), but the mechanical properties lag behind (bending of the sample is limited, not stretchable). The prototype can be seen in Figure 3 together with detail captured by the microscope with optical measurement of precision of the fabrication process. This foil is called flexible.

The second method uses only two materials, which are carbon powder (Vulcan XC72, Cabot) and silicon (Ecoflex 00-30, Smooth-On). Material for electrodes must be stretchable as the silicon. This is achieved by mixing the carbon powder and silicon in the weight ratio of $1: 5$, respectively [5]. Electrodes are patterned on a thin layer of silicon using a simple method of stamping. The stamp was fabricated by 3D printing using PLA material. The final step is to cover the electrodes by a layer of silicon.

This foil has worse electrical properties than the previous one (lower breakdown voltage, critical electric field $\left.E_{\mathrm{c}}=15 \mathrm{kV} \cdot \mathrm{mm}^{-1}\right)$. On the other hand, mechanical properties of this foil overtake the mechanical properties of the flexible foil (stretchable, able to withstand relative extension up to $200 \%$ ) [5]. The big advantage of this foil is that both electrodes and insulation layer consist mainly of silicon. This creates strong binding between them. Then, the entire foil acts as one material even during high mechanical extension. This foil is called stretchable.

1 AJP is a selective deposition technology based on generating and printing the aerosol. The aerosol is generated in devices called atomizers - pneumatic atomizer (used in the experiment) and ultrasonic atomizer. The aerosol is then transported by nitrogen flow to the printing nozzle. Another flow of nitrogen focuses the aerosol stream in the printing head.

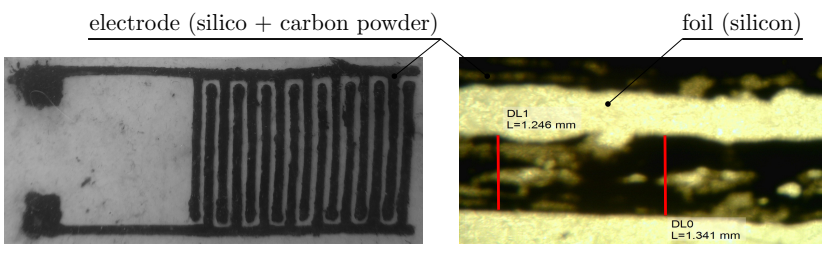

Figure 4: First prototype of elastic electroadhesive foil (left figure shows fabricated foil, right figure shows details captured by microscope)
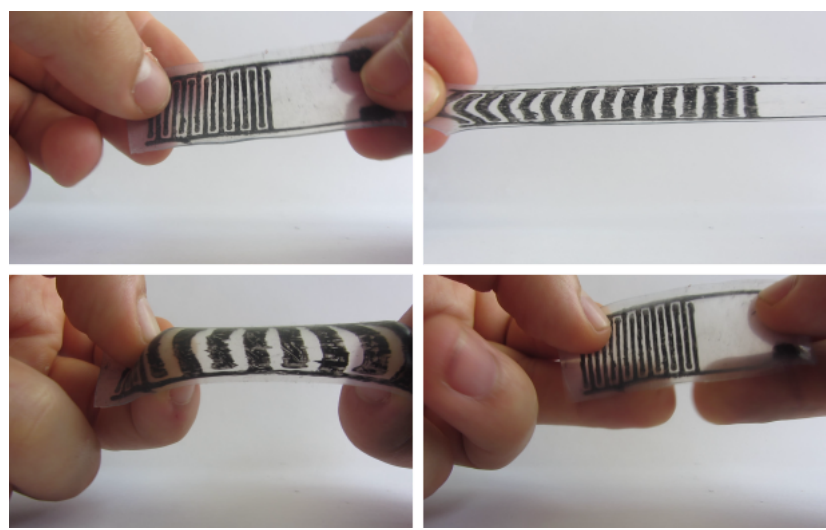

Figure 5: Demonstration of mechanical properties of elastic electroadhesive foil

The prototype of elastic electroadhesive foil can be seen in Figure 4 with details captured by microscope with optical measurement of the fabrication precision (scale of the detail in Figure 3 and 4 is the same). It should be noted that the prototype was not fabricated by a machine production and therefore precision and final size of the foil can be significantly improved. Finally, mechanical properties of fabricated foil are shown Figure 5.

\section{Formulation of mathematical model}

The distribution of electric field $\boldsymbol{E}$ within the general electrode system follows from the equation

$$
\operatorname{div} \varepsilon \operatorname{grad} \varphi=0,
$$

where $\varepsilon$ denotes the permittivity, $\varphi$ is scalar electric potential $(\boldsymbol{E}=-\operatorname{grad} \varphi)$. Externally generated volume charge density is neglected (attracted material is not previously polarised).

To get results from numerical solution of (1) there is no need to solve the whole surface where the attraction force $\boldsymbol{F}_{\text {a }}$ occurs. The electric field $\boldsymbol{E}$ can be calculated only in one segment that consists of two electrodes with oppo- 
site charges. The reason is a repetitive pattern of electrodes [6].

The electrostatic force $\boldsymbol{F}_{\mathrm{e}}$ exerted on the segment can be calculated from the distribution of $\boldsymbol{E}$ using the formula

$$
\boldsymbol{F}_{\mathrm{e}}=\oint_{S} \boldsymbol{T} \mathrm{d} \boldsymbol{S}, \boldsymbol{T}=\frac{1}{2}(\boldsymbol{E} \cdot \boldsymbol{D}) \boldsymbol{I}+\boldsymbol{E} \otimes \boldsymbol{D},
$$

where $\boldsymbol{T}$ is the Maxwell stress tensor ( $S$ being the outward normal to complete boundary of the attracted object). Symbol $\boldsymbol{D}$ represents the dielectric flux density $(\boldsymbol{D}=$ $\varepsilon \boldsymbol{E}), \boldsymbol{I}$ is unit diagonal matrix and symbol $\otimes$ denotes the dyadic product [6].

Numerical calculation of (2) can be simplified assuming interdigital electrodes parallel with the surface of attracted object. The Maxwell stress tensor can be then represented as follows

$$
\boldsymbol{T}=\left[\begin{array}{cc}
\frac{\varepsilon}{2}\left(E_{x}^{2}-E_{y}^{2}\right) & \left.\varepsilon E_{x} E_{y}\right) \\
\left(\varepsilon E_{y} E_{x}\right) & \frac{\varepsilon}{2}\left(E_{y}^{2}-E_{x}^{2}\right)
\end{array}\right],
$$

where $E_{x}$ and $E_{y}$ are electric field components. Normal direction of electrostatic force $F_{\mathrm{ey}}$ acting on the segment with electrodes of length $l$ is then given

$$
F_{\mathrm{e} y}=\oint_{S} T_{y} \mathrm{~d} \boldsymbol{S}=\frac{1}{2} \varepsilon l \int_{0}^{w+g}\left(E_{y}^{2}-E_{x}^{2}\right) \mathrm{d} x .
$$

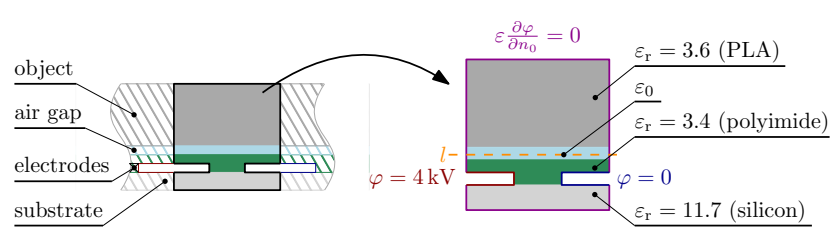

Figure 6: Definition area of electroadhesion foil (values of model parameters correspond with fabricated and measured foil)

Let us also mention often used [3] but also rough calculation approach that yields the electrostatic force $F_{\mathrm{e} y}$ between the two parallel plates of a capacitor

$$
F_{\mathrm{e} y}=-\frac{\partial W_{\mathrm{e}}}{\partial y}=-\frac{1}{2} \frac{\partial E_{y} D_{y}}{\partial y} \int_{V} \mathrm{~d} V=-\frac{\varepsilon}{2}\left(\frac{U}{t}\right)^{2} w l,
$$

where $W_{\mathrm{e}}$ is total energy, $D_{y}$ is electric displacement field component, $V$ is volume of capacitor, $U$ is applied voltage and $t$ is distance between electrodes.

\section{Results and discussions}

Design analysis of electroadhesive foil was performed by numerical solution of formulated mathematical model

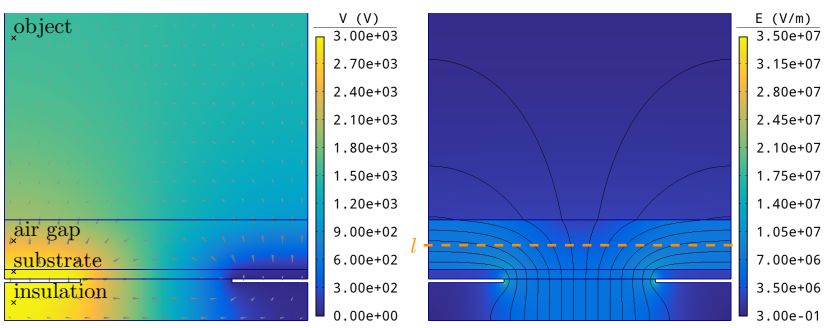

Figure 7: Distribution of scalar electric potential $\varphi$ (left figure) and electric field $\boldsymbol{E}$ (right figure) in the area of electroadhesive foil, air gap and object
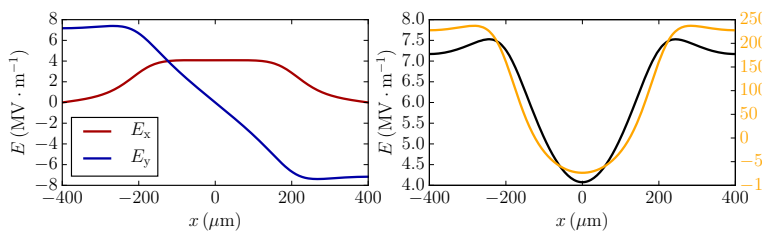

Figure 8: Electric field components $E_{x}, E_{y}$ (left figure), electric field $E$ and Mawell stress tensor component $\frac{\varepsilon}{2}\left(E_{y}^{2}-E_{x}^{2}\right)$ (right figure) in the air gap

(COMSOL Multiphysics and Agros2D was used). Figure 7 shows distribution of scalar electric potential $\varphi$ and electric field $\boldsymbol{E}$ in the definition area.

Figure 8 (left figure) shows that on the edge of each electrodes is peak of Maxwell stress tensor component function. Then, the function decreased in the direction to segments axis and reaches minimum there. In opposite direction the function decreased slightly until it reaches electrodes axis and then it rises back to its maximum.

From the experiments, attractive force for $U=3000 \mathrm{~V}$ calculated by (2) is $F_{\mathrm{a}}=0.0135 \mathrm{~N}$ (Eggshell method), by (3) $F_{\mathrm{a}}=0.0134 \mathrm{~N}$ and finally by (4) $F_{\mathrm{a}}=0.4 \mathrm{~N}$. Results are comparable with measurement $F_{\mathrm{a}}=0.0147 \mathrm{~N}$.

Parameters of the electroadhesive foil with interdigital electrodes are depicted in Figure 9 (bottom left). Figure 9 (top left) shows dependence of electroadhesion force $F_{\text {a }}$ on the air gap $d$. As can be seen, imperfect contact between the electroadhesive foil and the manipulated object is of huge importance. This implies usage of materials that are strongly stretchable. Influence of air gap can be also slightly reduced by a higher value of applied voltage $U$ but materials with high breakdown voltage have to be used (Figure 9 top left).

Dependencies of electroadhesion force $F_{\mathrm{a}}$ on the thickness of the insulation layer $t$ and height of that manipulated object $h$ are shown in Figure 9 (top right). With higher thickness $t$, the electroadhesive force rapidly decreases. This also implies usage of insulation materials with high breakdown voltage. If the manipulated object is 

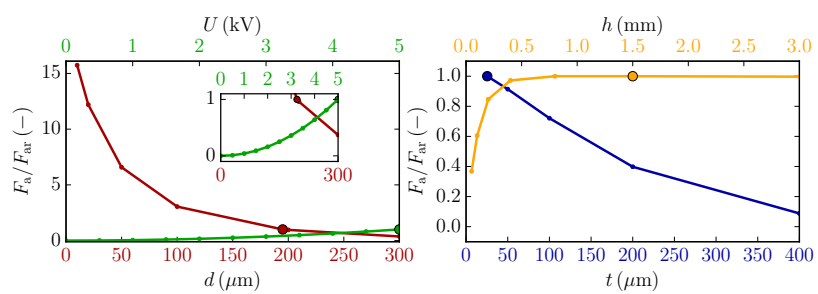

Figure 9: Dependency of electroadhesion force on parameter $d$ (red) and $U$ (green) (left figure), $t$ (blue) and $h$ (yellow) (right figure)

under $1 \mathrm{~mm}$ thick, the adhesion can be reduced to $50 \%$ of its full potential. This limits the usage of the electroadhesion to operate on thin films.

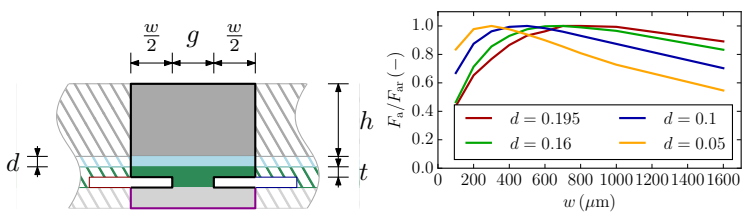

Figure 10: Optimization parameters on electroadhesive foil (left figure) and optimization curves of force $F_{\mathrm{a}}$ (right figure)

With fixed width of gap between electrodes $g$ (which is limited by insulation material) ideal width of electrodes $w$ exists (see Figure 9, left). Even though, widening of electrodes increases the electroadhesion force $F_{\mathrm{a}}$ on one segment, this increase cannot compensate the increase in area. This is also true in the opposite direction. If we decrease the width of electrodes $w$ under the optimum width, the saving in length of the segment does not compensate the decrease in the electroadhesion force. The optimum width of electrodes is dependent on air gap $d$. With thicker air gap the electrode must be wider, because higher values of the electric field are needed.

Experimental measurements were done to verify the results of numerical analysis using the electroadhesive foil manufactured by AJP. Figure 11 shows experimental setup for measurement of electrostatic force $F_{\mathrm{e}}$ (horizontal position of the foil minimize adhesion which is not reflected

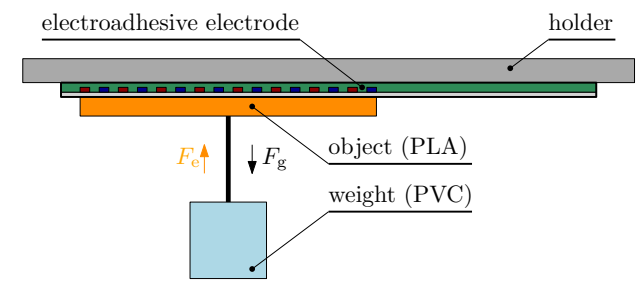

Figure 11: Arrangement for experimetnal verification
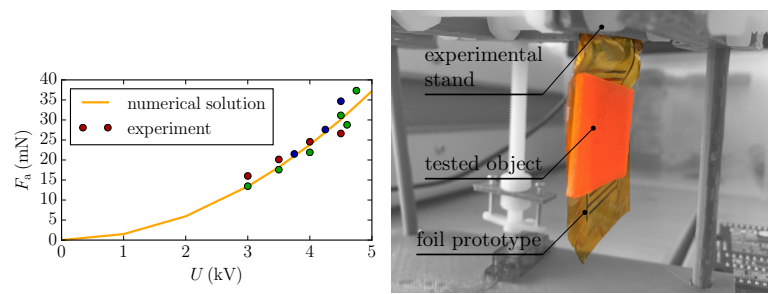

Figure 12: Comparing of experimental measurement and numerical analysis (left figure) and experimental testing of fabricated prototype of electroadhesive foil (right figure)

in the formulated mathematical model). Electrostatic force $F_{\mathrm{e}}$ was then measured $F_{\mathrm{e}}=F_{\mathrm{g}}$.

Figure 12 (left) shows results of the measurement and comparison with numerical analysis. The points represent the actual electrostatic forces $F_{\mathrm{e}}$ that were measured. It can be seen that points correspond with the trend of the curve and small differences are caused by air gap $d$ which cannot be measured.

Finally, Figure 12 (right) shows experimental testing of the electroadhesion prototype (vertical position is shown for better visualization). Experiments were done only with flexible foil. The reason for that is manufacturing precision for stretchable foil which did not meet desired requirements. The width of electrodes was not consistent and voltage breakdown occurred between two opposite electrodes. Therefore the electroadhesive phenomena cannot be seen.

\section{Conclusion}

The fabrication of the electroadhesive foil demands materials that are stretchable and materials with high breakdown voltage (insulation layer $t$ and air gap $d$ are of huge importance [7]). Materials are subject to future research.

Stretchable electroadhesive foil presented in this paper has big potential in this field of soft robotics. The mechanical properties are on very high level. There is a large space for improvements in electrical properties of silicon. Higher breakdown voltage must be obtained. This can be achieved by using dielectric powders as additives. The identical procedure was used to make conductive silicon by carbon powder. Future investigation for increasing the breakdown voltage of silicon must be made.

Another challenge is the manufacturing process of stretchable foil. Silicon and compounds containing silicon are very tricky to handle. Conventional fabricating method (Screen printing) fail when silicon is used, because of the high surface tension of silicon. This implies that new methods must be developed. Stamping presented in this pa- 
per appeared to be the most promising for fabrication of stretchable foil. It is very simple method with relatively high accuracy. As mentioned before there was no machine production and this is the biggest reason why the fabricated foil failed. Quality of patterned electrodes will be increased by using a precise machine production.

By using the most advanced materials and novel fabrication methods, devices using electrostatic electroadhesion effect will perform better and can be used in a variety of applications [8, 9]. One of the examples is space technology (inspection robots, docking system). The main benefits of electroadhesion are the simplicity of mechanism, cheap fabrication and a high ratio of foil weight to object that can handle (1: 10 was measured).

Acknowledgement: This research has been supported by the Ministry of Education, Youth and Sports of the Czech Republic under the RICE - New Technologies and Concepts for Smart Industrial Systems, project No. LO1607.

\section{References}

[1] Fessl J., Mach F., Navrátil J., Numerical and Experimental Analysis of Electrostatic Adhesion Force Generated by Interdigital Electrodes, 18th International Symposium on Electromagnetic Fields in Mechatronics, Electrical and Electronic Engineering, 2017
[2] Kuthan J., Mach F., Magnetically guided actuation of ferromagnetic bodies on the planar surfaces: Numerical modeling and experimental verication, 18th International Conference Computational Problems of Electrical Engineering, 2017

[3] Germann J., Dommer M., Pericet-Camara R., Floreano D., Active connection mechanism for soft modular robots, Advanced Robotics, 26.7, Taylor \& Francis, 2012

[4] Bamber T., Huo J., Singh J., Bigharaz M., Petzing J., Bingham P. A., Justham L., Penders J., Jackson M., Visualization methods for understanding the dynamic electroadhesion phenomenon, Journal of Physics D: Applied Physics, 50.20, 2017

[5] Germann J., Schubert B., Floreano D., Stretchable electroadhesion for soft robots, Intelligent Robots and Systems (IROS 2014), 2014 IEEE/RSJ International Conference on, 2014

[6] Cao C., Sun X., Fang Y., Qin Q., Yu A., Feng X., Theoretical model and design of electroadhesive pad with interdigitated electrodes, Materials \& Design, 89, Elsevier, 2016

[7] Huo J., Tailor M., Bamber T., Chamberlain M., Justhamand L., Jackson M., Investigation of relationship between interfacial electroadhesive force and surface texture, Journal of Physics D: Applied Physics, 49.3, 2015

[8] Chen R., Huang Y., Tang Q., An analytical model for electrostatic adhesive dynamics on dielectric substrates, Journal of Adhesion Science and Technology, 31.11, Elsevier, 2017

[9] Chen R., A gecko-inspired electroadhesive wall-climbing robot, IEEE Potentials, 34.2, 2015

[10] Prahlad H., Perline R., Stanford S., Marlow J., Kornbluh R., Electroadhesive robots - wall climbing robots enabled by a novel, robust, and electrically controllable adhesion technology, Robotics and Automation (ICRA), IEEE International Conference on, 2008 